\title{
Measurements of Tyre/Road Noise and of Acoustical Properties of Porous Road Surfaces
}

\author{
Malcolm J. Crocker ${ }^{\dagger}$ and Zhuang $\mathrm{Li}^{\dagger}$ \\ Department of Mechanical Engineering, Auburn University, Auburn, AL 36849, USA
}

\author{
Jorge P. Arenas ${ }^{\dagger}$ \\ Institute of Acoustics, University Austral of Chile, PO Box 567, Valdivia, Chile
}

(Received 29 April 2005; accepted 20 May 2005)

\begin{abstract}
The three main noise sources in modern cars and trucks are tyre/road interaction, the power plant, and the wind turbulence. Tyre/road interaction noise is receiving increasing attention. The main tyre/road interaction mechanisms consist of: 1) impacts between the tyre tread and the road, which cause radial, tangential, and sidewall tyre tread and carcass vibration and consequent noise radiation, and 2) air displacement mechanisms caused by the tyre, the major one probably being the so-called "air pumping" in, or between, the tyre tread and the road surface. In this paper, the results of tyre/road interaction noise measured on different types of road pavement surfaces are presented. The results were obtained by means of the close proximity method. The sound absorption coefficients of dense and porous road surfaces were measured in the Sound and Vibration Research Laboratory at Auburn University 1) using core samples with 102 and $153 \mathrm{~mm}$ diameter impedance tubes and 2) with the same two impedance tubes mounted vertically on pavement slabs with surfaces similar to those of the core samples. In addition, the sound absorption coefficient of road surfaces of similar type to those of the cores and slabs was measured in situ, also with the tubes mounted vertically. The peak sound absorption coefficient measured of the fine and coarse mix aggregate porous surfaces suggests that the first peak frequency and peak absorption coefficient magnitude is only slightly different for the two types of porous surfaces. Since the fine mix aggregate porous surface is smoother, it is preferred since it should result in less tyre tread impact noise and thus lower overall tyre noise than the coarse aggregate surface. A porous surface of between 38 and $50 \mathrm{~mm}$ thickness is recommended for the type of porous surface examined, if a peak absorption frequency of about $1000 \mathrm{~Hz}$ is desired. Such a surface would be most effective at reducing interstate highway noise of automobiles. In addition, porous road pavement surfaces have the further advantage that they drain water well and reduce the splash up behind vehicles during heavy rainfalls.
\end{abstract}

${ }^{\dagger}$ Member of the International Institute of Acoustics and Vibration (IIAV)

\section{INTRODUCTION}

\subsection{Sources of Noise on Cars and Trucks}

The three main noise sources in modern cars and trucks are tyre/road interaction, wind turbulence, and the power plant. ${ }^{1}$ The power unit noise is composed of contributions from the fan, engine, exhaust, and transmission. In addition, the engine noise contains contributions from the injection system, intake, and cylinder block. ${ }^{1}$ Noise is of course a problem both inside the passenger compartments (interior noise) of vehicles and in residential areas near to the highway on which the vehicles are traveling (exterior noise). In most modern automobiles, the engine and exhaust noise have been sufficiently suppressed so that they are no longer the dominant interior or exterior noise sources. On heavy trucks, the engine is normally a diesel, and the suppression of its noise is more difficult.

With vehicle noise, regulations are mostly concerned with exterior noise of vehicles, and this paper will consider mostly the exterior noise. Of course, if the exterior noise is reduced, the interior noise is normally reduced as well, although the relationship may not be quite linear. With modern well designed automobiles, the exterior noise is dominated by tyre noise at almost all speeds, even under accelerating conditions, except for acceleration in the first and second gears. With trucks, however, at speeds up to about $70 \mathrm{~km} / \mathrm{h}$, engine and exhaust noise is dominant under accelerating conditions. But above this speed, tyre noise becomes equally as important as engine, exhaust, and other power unit related noise sources, particularly as far as the exterior noise is concerned. ${ }^{2}$ This fact has been observed experimentally, and it has been found that similar trends exist for the exterior noise of a wide variety of manufactured vehicles and that the exterior noise of automobiles is significantly less than that of heavy trucks by almost $15 \mathrm{~dB}$ at low speed and by almost $10 \mathrm{~dB}$ at medium to high speed. ${ }^{1}$ One can conclude from the previous discussion that much of the exterior noise emitted from modern cars and trucks, particularly at medium to high speeds, is caused by tyre/road interaction noise.

Of course, the frequency content of noise as well as its magnitude is very important. It has been observed that for automobiles travelling at medium to high speeds, the A-weighted noise peaks at about $1000 \mathrm{~Hz}$, while for light and heavy trucks, the annoying noise peaks at a frequency closer to 1200 to $1600 \mathrm{~Hz}^{2}$

In addition, it has been shown that the A-weighted exterior sound levels emitted by passenger cars and light and heavy trucks has not changed very much over time. ${ }^{2}$ This is probably because the power of automobiles has increased in many countries, particularly in the USA, and it is has been 